\title{
Do not skip a beat: intermittent Wolff-Parkinson-White syndrome diagnosed by a single beat on a 12-lead ECG
}

\author{
Mohammed Abrahim ${ }^{1,4}(1) \cdot$ Amal Mattu $^{2} \cdot$ Jan Grzeslo ${ }^{1}$ Janna AbdelAziz ${ }^{3}$ \\ Received: 10 September 2020 / Accepted: 20 October 2020 / Published online: 4 January 2021 \\ (c) Canadian Association of Emergency Physicians (CAEP) / Association Canadienne de Médecine d'Urgence (ACMU) 2020
}

Keywords Wolff-Parkinson-White syndrome · Adolescent palpitations · Arrhythmias · Dysrhythmias · Intermittent WPW syndrome $\cdot$ Syncope

\section{Introduction}

Wolff-Parkinson-White syndrome is characterized by electrical impulse conduction via an accessory pathway between the atria and ventricles which bypasses the physiological delay of the $\mathrm{A}-\mathrm{V}$ node [1]. The three main electrocardiogram (ECG) features of Wolff-Parkinson-White syndrome are a short PR interval $(<0.12 \mathrm{~s})$, a prolonged QRS complex $(>0.10 \mathrm{~s}$ ), and a slurred onset of the QRS complex (delta wave) [2].

Wolff-Parkinson-White syndrome is, in most cases, an asymptomatic condition. However, sudden cardiac death can be the first presentation of Wolff-Parkinson-White syndrome and the overall risk of sudden cardiac death in Wolff-Parkinson-White syndrome is estimated at $0.1 \%$ in asymptomatic patients and $0.3 \%$ in symptomatic patients per year [3]. Wolff-Parkinson-White syndrome accounts for $1.6 \%$ of all sudden cardiac death among young athletes in the USA [4]. Early diagnosis of Wolff-Parkinson-White or other accessory pathways can lead to timely catheter ablation which is now considered a safe and curative procedure [5].

Mohammed Abrahim

mabrahim@haltonhealthcare.com

1 Department of Family Medicine, McMaster University, Hamilton, ON L8S 4L8, Canada

2 Department of Emergency Medicine, University of Maryland School of Medicine, Baltimore, MD 21201, USA

3 Department of Health Sciences, Carleton University, Ottawa, ON K1S 5B6, Canada

4 Emergency Department, Milton District Hospital, 725 Bronte Street South, Milton, ON L9T 9K1, Canada

\section{Presentation}

A previously healthy 15 -year-old female patient presented to the emergency department (ED) with episodes of palpitations which had been occurring intermittently over the prior few months and had become more frequent during the past few weeks. Prior to her ED visit, she sought medical advice through her primary care physician who diagnosed her, without cardiac workup, with anxiety, likely related to her schoolwork. In the ED, she was experiencing intermittent palpitations at triage where she received a 12-lead ECG (Fig. 1) showing evidence of Wolff-Parkinson-White syndrome (short PR interval, delta wave, slight widening of the QRS complex) in a single P-QRS complex. Her physical examination was normal. Her laboratory investigations were all non-diagnostic.

She was placed on a cardiac monitor. While in the examination room being seen by the emergency physician, her palpitations became persistent. The cardiac monitor demonstrated a regular narrow complex tachycardia. A second ECG was done (Fig. 2) and demonstrated a supraventricular tachyarrhythmia. Adenosine $6 \mathrm{mg}$ intravenous push was given and resulted in restoration of normal sinus rhythm (Fig. 3). Notably, the signs of Wolff-Parkinson-White syndrome had resolved as well. She was initiated on an oral beta-blocker and referred to the electrophysiology services for consideration of ablation procedure.

\section{Impression}

A 15-year-old female with newly diagnosed intermittent Wolff-Parkinson-White syndrome. The pathognomonic ECG features were captured in a single beat on a 12-lead 
Fig. 1 12-lead ECG arrows pointing towards the delta waves in a single beat

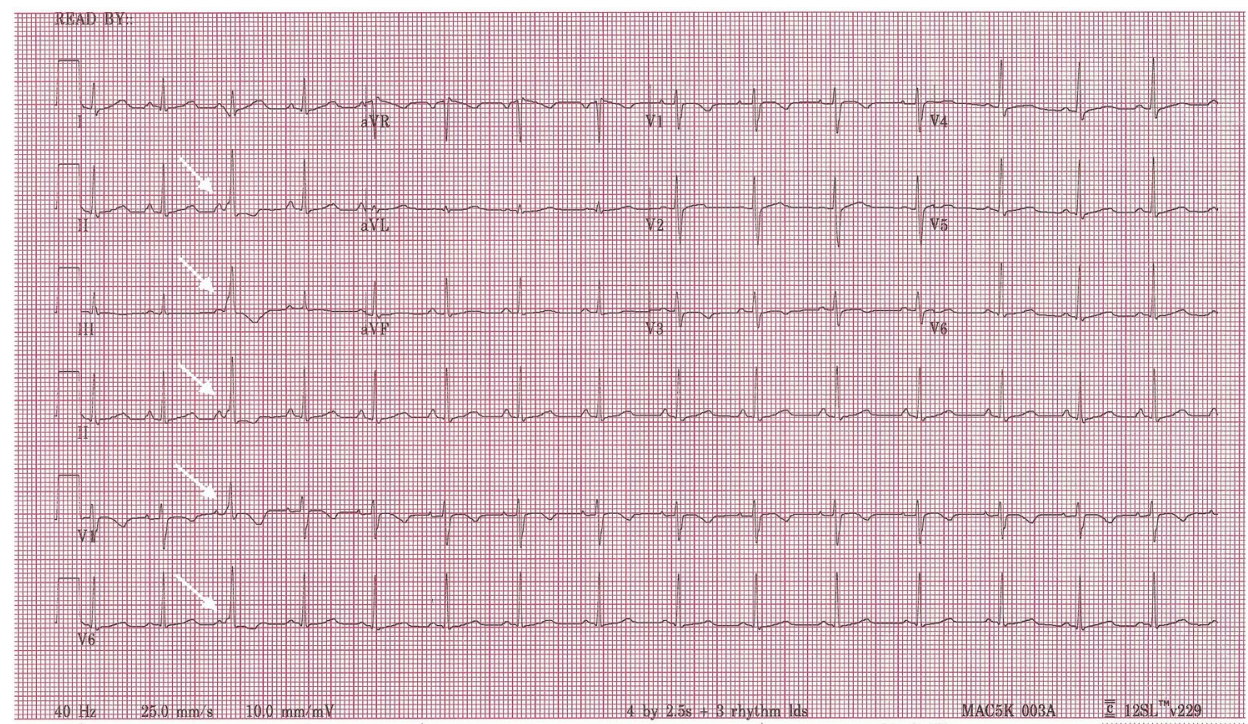

ECG performed during triage. Those features were absent on the subsequent ECGs.

\section{Conclusion}

Diagnosis of Wolff-Parkinson-White syndrome should be considered in the differential diagnosis for patients of all ages with palpitations or objective signs of dysrhythmias. Further to this, physicians should have a low threshold in performing a 12-lead ECG for all patients with palpitations in the ED. Cardiac and non-cardiac aetiology of palpitations must be ruled out first before diagnosing psychological causes (anxiety/panic disorder) and anxiety-induced palpitations should remain as a diagnosis of exclusion. Sudden cardiac death can be the first presentation of Wolff-Parkinson-White and the risk of sudden cardiac death triples in symptomatic patients. Emergency physicians should be liberal in ordering triage and repeat ECGs for patients presenting with palpitations, especially when they become symptomatic. Every beat in the ECG could carry the diagnosis - do not skip a beat!
Fig. 2 12-lead ECG showing supraventricular tachycardia (SVT)

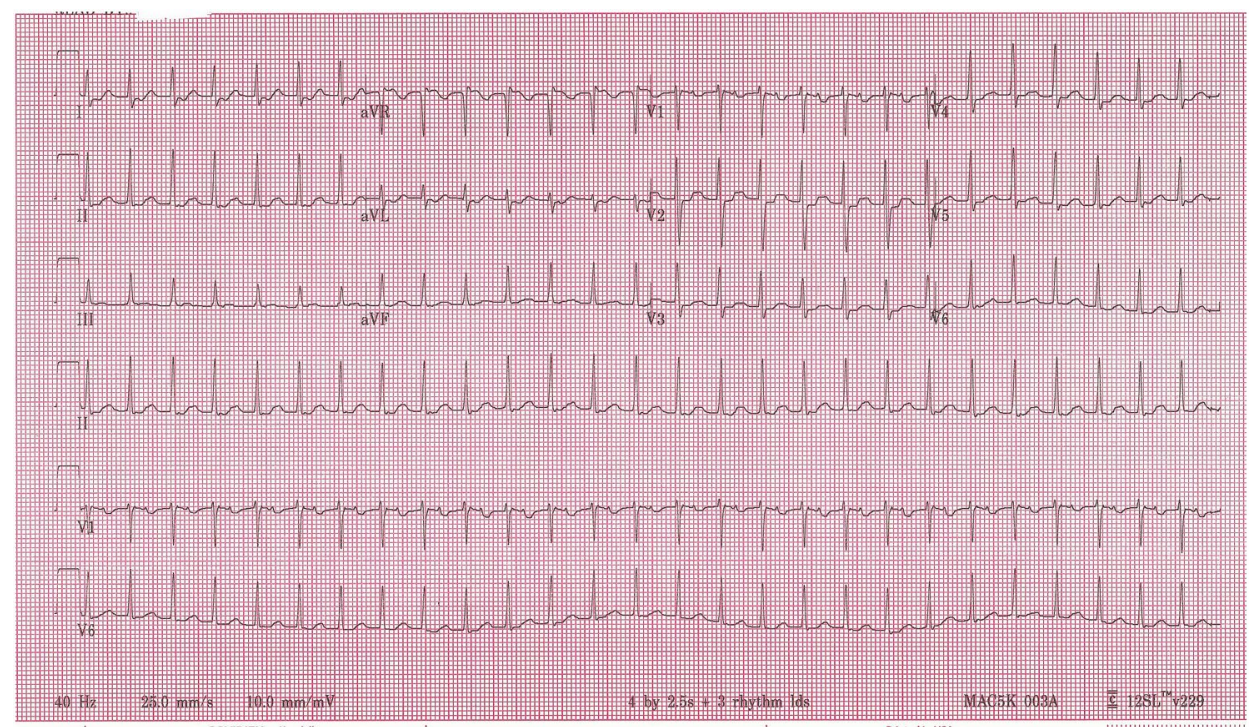


Fig. 3 12-lead ECG post-adenosine $6 \mathrm{mg}$ IV push with resolution of delta waves and SVT

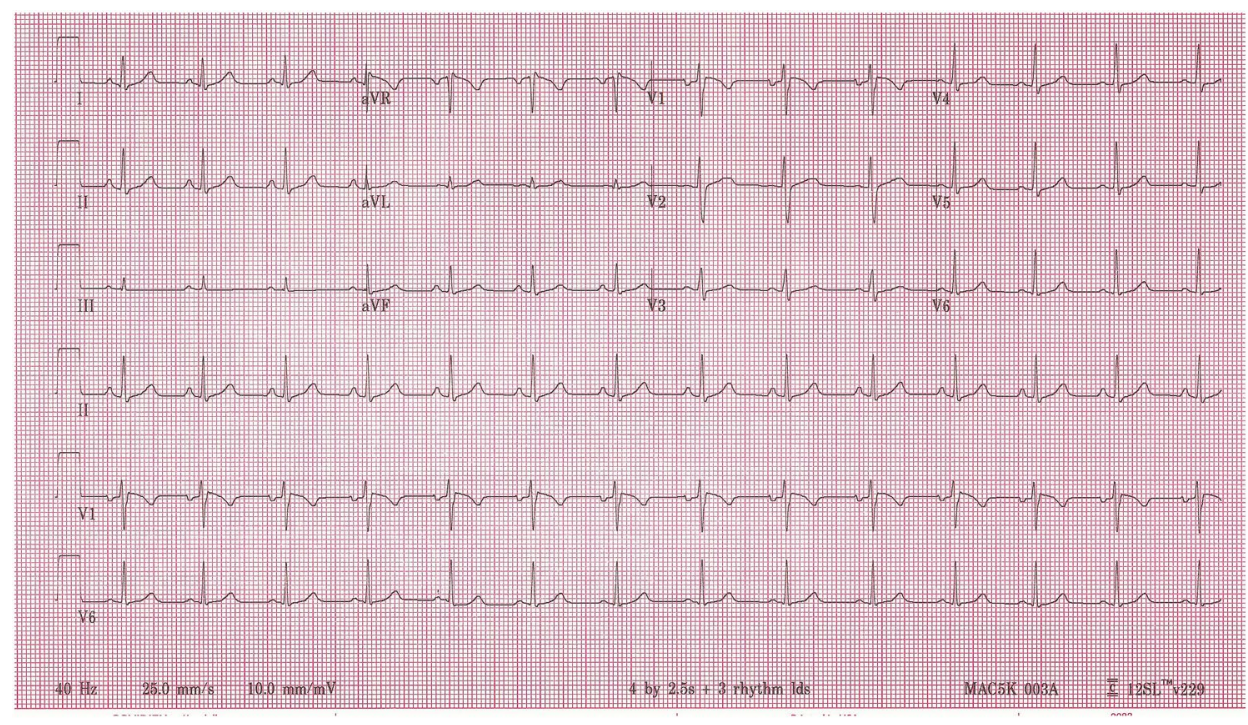

Funding None.

\section{Compliance with ethical standards}

Conflict of interest The authors declare that they have no conflict of interest.

\section{References}

1. Al-Khatib SM, Pritchett EL. Clinical features of Wolff-Parkinson-White syndrome. Am Heart J. 1999;138(3):403-13.

2. Segers M, Lequime J, Denolin H. L'activation ventriculaire précoce de certains cœurs hyperexeitables. Cardiology. 1944;8(3-4):113-67.

3. Laaouaj J, Jacques F, O'Hara G, Champagne J, Sarrazin JF, Nault I, Philippon F. Wolff-Parkinson-White as a bystander in a patient with aborted sudden cardiac death. HeartRhythm Case Rep. 2016;2(5):399.

4. Maron BJ, Doerer JJ, Haas TS, Tierney DM, Mueller FO. Sudden deaths in young competitive athletes. Circulation. 2009;119(8):1085-92.

5. Cohen MI, Triedman JK, Cannon BC, Davis AM, Drago F, Janousek J, Klein GJ, Law IH, Morady FJ, Paul T, Perry JC. PACES/HRS expert consensus statement on the management of the asymptomatic young patient with a Wolff-Parkinson-White (WPW, ventricular preexcitation) electrocardiographic pattern: developed in partnership between the Pediatric and Congenital Electrophysiology Society (PACES) and the Heart Rhythm Society (HRS). Endorsed by the governing bodies of PACES, HRS, the American College of Cardiology Foundation (ACCF), the American Heart Association (AHA), the American Academy of Pediatrics (AAP), and the Canadian Heart Rhythm Society (CHRS). Heart Rhythm. 2012;9(6):1006-24. 